\title{
Texton Based Image Retrieval Using Indexed LBP Transitions
}

\author{
A. Srinivasa Rao, V.Venkata Krishna, Prof.YKSundara Krishna \\ Research Scholoar ,Krishna University, Machilipatnam, Krishna (dt),AP,INDIA \\ akella.srinivas08@gmail.com \\ Professor in CSE Dept.GIET,Rajahmundry,Andhra Pradesh, INDIA \\ vakula.krishna@gmail.com \\ Professor in CSE Dept. Krishna University, Machilipatnam, Andhra Pradesh, INDIA
}

\section{ABSTRACT}

yksk2010@gmail.com

The present paper derived a new model of texture image retrieval by integrating the transitions on Local Binary Pattern (LBP) with textons and Grey Level Co-occurrence Matrix (GLCM). The present paper initially derived transitions that occur from 0 to 1 or 1 to 0 in circular manner on LBP. The transitions reduce the 256 LBP codes into five texture features. This reduces the lot of complexity. The LBP codes are rotationally variant. The proposed circular transitions on LBP are rotationally invariant. Textons, which represents the local relationships,are detected on this. The GLCM features are evaluated on the texton based image for efficient image retrieval. The proposed method is experimented on a huge data base of textures collected from Google data base. The experimental result indicates the efficiency of the proposed model.

Keywords: Image retrieval; rotational invariant; textons; GLCM.

\section{Council for Innovative Research}

Peer Review Research Publishing System

Journal: INTERNATIONAL JOURNAL OF COMPUTERS \& TECHNOLOGY

Vol. 13, No.12

www.ijctonline.com, editorijctonline@gmail.com 


\section{INTRODUCTION}

The twentieth century has witnessed unparalleled growth in the number, availability and importance of images in all walks of life. Images now play a crucial role in fields as diverse as medicine, journalism, advertising, design, education, entertainment, etc. Technology, in the form of inventions such as photography and television, has played a major role in facilitating the capture and communication of image data. Problems with traditional methods of image indexing [1] have led to the rise of interest in techniques for retrieving images on the basis of automatically derived features such as color, texture and shape in a technology now generally referred to as Content Based Image Retrieval (CBIR). The CBIR systems allow the user to iteratively search image databases looking for those images which are similar to a specified query image. Selection and ranking of relevant images from image collections remains a problem in CBIR. Image retrieval has been an active research topic in recent years due to its potentially large impact on both image understanding and Web image search.

\subsection{Content Based Image Retrieval (CBIR) Systems}

The earliest use of the term CBIR in the literature seems to have been by Kato [2], to describe his experiments into automatic retrieval of images from a database by color and shape feature. The features used for retrieval can be either primitive or semantic, but the extraction process must be predominantly automatic. CBIR draws many of its methods from the field of image processing and computer vision, and is regarded by scholars as a subset of that field. There has been a lot of interest in content-based image retrieval using visual features over the last decade. An overview of work in this area can be found in $[3,4,5,6,7,8,9]$. CBIR is like an information filter process and is expected to provide a high percentage of relevant images in response to user query [10]. The presence of large volumes of digital repositories leads to many schemes of indexing and retrieval of such data (e.g.,QBIC [11, 12], Netra [13], VisualSeek [14], Chabot [15], Blobworld [16], photobook [17], etc). In all these cases, the user is interested in seeking the most similar images to his query.

Gudivada et al. [18] have identified many important applications of general purpose CBIR. The CBIR has been applied for many purposes[19] in law enforcement and crime prevention, such as fingerprint recognition [20], face recognition [21], DNA matching, shoe sole impressions [22], and surveillance systems [23]. Integrated tools in Web image retrieval can help considerably in identifying suspicious sites and thus in filtering them.

Color is one of the most important image indexing features employed in CBIR. Schettini et al. [24] and Del Bimbo [3] provide a comprehensive survey of various methods employed for color image indexing and retrieval in image databases. Some of the popular methods to characterize color information in images are color histograms [25, 26], color moments [27], and color correlograms [28]. Though all these methods provide good characterization of color, they have the problem of high-dimensionality. This leads to more computational time, inefficient indexing, and low performance. To overcome these problems, use of SVD [25], dominant color regions approach [29, 30], and color clustering [31, 32] have been proposed. Many authors have done a survey of various shape methods used for content-based image retrieval [3, 33, 9 , 34]. Recently, techniques using shape measure as an important feature have been used for CBIR.

The texture features are one of the important features for an efficient and cost effective retrieval system. Many texture features derived from various approaches of texture analysis and classification are thus important and crucial to build an efficient retrieval system[10, 35, 36, 37, 38, 39, 40, 41, 42, 43, 44, 45,46].

The rest of the paper is organised as follows. Section 2 describes the proposed texton based transitions LBP. Experimental results are discussed in section 3 and conclusions are given in section 4.

\section{METHODOLOGY}

Proposed Texton basedTransitioned LBP (TTLBP) model:

The proposed TTLBP model aims to overcome rotational variance problem and to reduce the large number of codes that are derived by LBP. The proposed TTLBP model consists of five steps as given below.

\section{Step 1: Color Quantization of 7-bit Binary Code.}

During the course of feature extraction, the original images are quantized into 128 colors of RGB color space and the color gradient is computed from the RGB color space and then the statistical information of textons is calculated to describe image features. In order to extract gray level features from color information, the proposed TTLBP model utilized the RGB color space which quantizes the color space into 7-bins to obtain 128 gray levels. The index matrix of 128 color image is denoted as $\mathrm{C}(\mathrm{x}, \mathrm{y})$. The RGB quantization process is done by using 7 -bit binary code of 128 colors as given in Eqn.(1).

$C(x, y)=16^{*} \mid(R)+2^{*} I(G)+I(B)$

where

$I(R)=0,0 \leq R \leq 16, \quad I(R)=i,\left(\left(16^{*} i\right)+1\right) \leq R \leq\left(16^{*}(i+1)\right)$

$$
\mathrm{i}=[1,2,3 \text {...7] }
$$

$I(G)=0,0 \leq G \leq 16, \quad I(G)=i,\left(\left(16^{*} i\right)+1\right) \leq G \leq\left(16^{*}(i+1)\right)$ 


$$
\mathrm{i}=[1,2 . .6 \quad]
$$

$l(B)=0,0 \leq B \leq 32, \quad I(B)=i,\left(\left(32^{*} i\right)+1\right) \leq B \leq\left(32^{*}(i+1)\right)$

$$
\mathrm{i}=[1,2,3]
$$

Therefore, each value of $\mathrm{C}(\mathrm{x}, \mathrm{y})$ is a 7 bit binary code ranging from 0 to 127 .

STEP 2:Representation of the Texture image by the number of circular transitions from 0 to 1 or 1 to 0 on LBP instead of LBP codes or numbers.

\subsection{Representation of LBP}

LBP is a gray-scale invariant texture measure computed from the analysis of a $3 \times 3$ local neighborhood over a central pixel. The LBP is based on a binary code describing the local texture pattern. LBP is a local texture operator with low computational complexity and low sensitivity to changes in illumination. LBP has the following advantages i) the local texture character can be described efficiently ii) it is easy to understand and compute iii) the whole image character description can be easily extended.LBP also suffers with following disadvantages i) in the course of analysis, its window size is fixed ii) It neglects the effect of the central pixel in local region iii) LBP code is rotationally variant.

In a square-raster digital image with a $3 \times$ neighborhood, each pixel is surrounded by eight neighboring pixels and this represents the smallest complete unit (in the sense of having eight directions surrounding the pixel). A neighborhood of $3 \times 3$ pixels is denoted by a set containing nine elements: $P=\left\{P_{0}, P_{1} \ldots P_{8}\right\}$, here $P_{0}$ represents the intensity value of the central pixel and $P_{i}\{i=1,2 \ldots 8\}$, is the intensity value of the neighboring pixel $i$. The eight neighbors are labeled using a binary code $\{0,1\}$ obtained by comparing their values to the central pixel value. If the tested gray value is below the gray value of the central pixel, then it is labeled 0 , otherwise it is assigned the value 1 as described by the Equation 5

$$
P_{i}=\left\{\begin{array}{c}
0 \quad P_{0}<P_{i} \\
1 \text { otherwise }
\end{array}\right.
$$

$\mathrm{P}_{i}$ is the obtained binary code, $P_{i}$ is the original pixel value at position $i$ and $P_{o}$ is the central pixel value. The Fig. 1 (a) shows the grey level values of a $3 \times 3$ neighborhood of an image. And the Fig. 1 (d) shows its corresponding binary labelling based on Equation 6 . The binary weights of the given $3 \times 3$ neighborhood are calculated by the Equation 5.2 .

$$
\text { Weights }_{L B P}=\sum_{i=0}^{7} P_{i} * 2^{i-1}(6)
$$

As each element of LBP has one of the two possible values, the combination of all the eight elements results in $2^{8}=$ 256 possible local binary patterns ranging from 0 to 255 . There is no unique way to label and order the 255 LBP on a $3 \times 3$ neighborhood.

\begin{tabular}{|l|l|l|}
\hline 63 & 28 & 45 \\
\hline 88 & 40 & 35 \\
\hline 67 & 40 & 21 \\
\hline
\end{tabular}

(a)

\begin{tabular}{|l|l|l|}
\hline $2^{0}$ & $2^{1}$ & $2^{2}$ \\
\hline $2^{\prime}$ & & $2^{3}$ \\
\hline $2^{6}$ & $2^{5}$ & $2^{4}$ \\
\hline
\end{tabular}

(c)

\begin{tabular}{|l|l|l|}
\hline 1 & 0 & 1 \\
\hline 1 & & 0 \\
\hline 1 & 1 & 0 \\
\hline
\end{tabular}

(b)

$1^{*} 2^{0}+0 * 2^{1}+1^{*} 2^{2}+0^{*} 2^{3}+$

$0 * 2^{4}+1 * 2^{5}+1^{*} 2^{6}+1^{*} 2^{7=} 229$

(d)

Fig. 1: (a) Sample Grey level Neighborhood (b) Conversion of Fig. 1 (a)into LBP (c) Representation of LBP-Weights (d) LBP code or LBP number.

Fig. 1 shows an example on how to compute LBP. The original $3 \times 3$ neighborhood is given in Fig. 1 (a). The central pixel value is used as a threshold in order to assign a binary value to its neighbors. Fig. 1 (b) shows the result of thresholding the $3 \times 3$ neighborhood. The obtained values are multiplied by their corresponding weights as shown by Fig. 1 (c). The LBP code is given in Fig.1 (d). which is 229 in this case the central pixel 40 is replaced by the obtained LBP value 229. In this way a new LBP image is constructed by processing each pixel and its $3 \times 3$ neighbors in the original image. The binary weights of Fig.1 (c) can be given in eight different ways as shown in Fig.2. 


\begin{tabular}{|l|l|l|}
\hline $2^{0}$ & $2^{1}$ & $2^{2}$ \\
\hline $2^{7}$ & & $2^{3}$ \\
\hline $2^{6}$ & $2^{5}$ & $2^{4}$ \\
\hline
\end{tabular}

(a)

\begin{tabular}{|l|l|l|}
\hline $2^{6}$ & $2^{7}$ & $2^{0}$ \\
\hline $2^{5}$ & & $2^{1}$ \\
\hline $2^{4}$ & $2^{3}$ & $2^{2}$ \\
\hline
\end{tabular}

(c)

\begin{tabular}{|l|l|l|}
\hline $2^{4}$ & $2^{5}$ & $2^{6}$ \\
\hline $2^{3}$ & & $2^{7}$ \\
\hline $2^{2}$ & $2^{1}$ & $2^{0}$ \\
\hline
\end{tabular}

(e)

\begin{tabular}{|l|l|l|}
\hline $2^{2}$ & $2^{3}$ & $2^{4}$ \\
\hline $2^{1}$ & & $2^{5}$ \\
\hline $2^{0}$ & $2^{7}$ & $2^{6}$ \\
\hline
\end{tabular}

(g)

\begin{tabular}{|l|l|l|}
\hline $2^{7}$ & $2^{0}$ & $2^{1}$ \\
\hline $2^{6}$ & & $2^{2}$ \\
\hline $2^{5}$ & $2^{4}$ & $2^{3}$ \\
\hline
\end{tabular}

(b)

\begin{tabular}{|l|l|l|}
\hline $2^{5}$ & $2^{6}$ & $2^{7}$ \\
\hline $2^{4}$ & & $2^{0}$ \\
\hline $2^{3}$ & $2^{2}$ & $2^{1}$ \\
\hline
\end{tabular}

(d)

\begin{tabular}{|l|l|l|}
\hline $2^{3}$ & $2^{4}$ & $2^{5}$ \\
\hline $2^{2}$ & & $2^{6}$ \\
\hline $2^{1}$ & $2^{0}$ & $2^{7}$ \\
\hline
\end{tabular}

(f)

\begin{tabular}{|l|l|l|}
\hline $2^{1}$ & $2^{2}$ & $2^{3}$ \\
\hline $2^{0}$ & & $2^{4}$ \\
\hline $2^{7}$ & $2^{6}$ & $2^{5}$ \\
\hline
\end{tabular}

(h)

Fig.2: Eight Different Ways of measuring LBP weights on a $3 \times 3$ neighborhood.

The value of the LBP changes by the representation of the weights. The LBP can be calculated in 8 different ways for a $3 \times 3$ neighborhood as shown in Fig. 5.2. That is for any $3 \times 3$ neighborhood one can generate eight LBP values. The LBP value for the Fig.1 (a) in all eight directions as represented in Fig.2 is given as 227, 242, 121, 188, 94, 47, 151, and 203 respectively.

To overcome the rotational variant disadvantage of LBP the proposed method measures the number of transitions from 0 to 1 or 1 to 0 in a circular manner and replaces the central pixel with the number of transitions. That is the above LBP 10100111 of Fig. 1 contains four transitions from 0 to 1 or 1 to 0 . The central pixel is replaced by the obtained number of transitions value 4 . In this way the second step derives a new LBP image by replacing each central pixel value of a $3 \times 3$ neighborhood with it's circular transition number that occur from 0 to 1 or 1 to 0 as shown in Fig.3.

\begin{tabular}{|l|l|l|}
\hline 163 & 228 & 45 \\
\hline 88 & 140 & 165 \\
\hline 167 & 48 & 21 \\
\hline
\end{tabular}

a) $3 \times 3$ neigh borhood

\begin{tabular}{|l|l|l|}
\hline 1 & 1 & 0 \\
\hline 0 & & 1 \\
\hline 1 & 0 & 0 \\
\hline
\end{tabular}

b) Generation of LBP

\section{0}
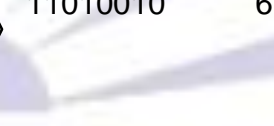

Fig.3: Derivation of circular transitions number on LBP.

The converted transitioned number LBP texture image pixels contain grey levels of $0,2,4,6$ and 8 only. This is because no circular LBP contains odd number of transitions and the number of circular transitions from 0 to 1 or 1 to 0 on 8 -neighboring LBP can be 2, 4, 6 or 8 only. The present paper indexed the above transition numbers $0,2,4,6$ and 8 as $1,2,3,4$ and 5 respectively.

Step-3: Texton detection ontransitioned number LBP texture image

The transitioned number LBP texture image of step2 generates an image with 5- indexed grelevel values i.e. 1,2,3,4 and 5. The present research evaluates Textons on these 5-grey level image in step3.

Textons $[47,48]$ are considered as texture primitives, which are located with certain placement rules. A close relationship can be obtained with image features such as shape, pattern, local distribution orientation, spatial distribution, etc.., using textons. Textons are effectively utilized to develop efficient models in the context of texture recognition or object recognition. Textons are considered as texture primitives which are positioned with certain placement rules. The textons are defined as a set of blobs or emergent patterns sharing a common property all over the image [47, 48]. The different 
textons may form various image features. To have a precise and accurate texture classification, the present study strongly believes that one need to consider the significant textons. There are several issues related with i) texton size ii) tonal difference between the size of neighbouring pixels iii) texton categories iv) expansion of textons in one orientation v) elongated elements of textons with jittered in orientation. By this sometimes a fine or coarse or an obvious shape may results or a pre-attentive discrimination is reduced or texton gradients at the texture boundaries may be increased. To achieve the above, the present paper utilized four texton types on a $2 \times 2$ grid as shown in Fig. 4 .

\begin{tabular}{|l|l|}
\hline$v_{1}$ & $v_{2}$ \\
\hline$v_{3}$ & $v_{4}$ \\
\hline
\end{tabular}

(a)

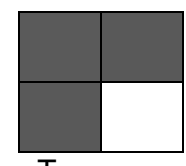

$\mathrm{T}_{4}$

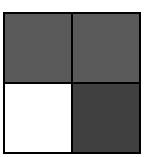

$\mathrm{T}_{1}$

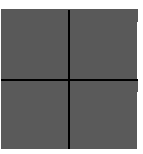

$\mathrm{T}_{5}$

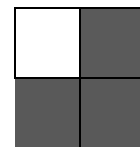

$\mathrm{T}_{2}$

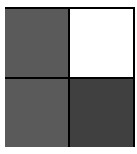

$\mathrm{T}_{3}$

Fig. 4 DifferentTextons representation on $2 \times 2$ grid.

c)

\begin{tabular}{|l|l|l|l|l|l|}
\hline 5 & 5 & 5 & 1 & 4 & 4 \\
\hline 5 & 2 & 2 & 2 & 0 & 4 \\
\hline 3 & 5 & 3 & 3 & 3 & 2 \\
\hline 3 & 5 & 5 & 3 & 2 & 2 \\
\hline 5 & 5 & 4 & 3 & 2 & 0 \\
\hline 5 & 1 & 4 & 1 & 3 & 3 \\
\hline 2 & 1 & 3 & 3 & 1 & 3 \\
\hline 2 & 2 & 3 & 4 & 1 & 1 \\
\hline
\end{tabular}

(a)

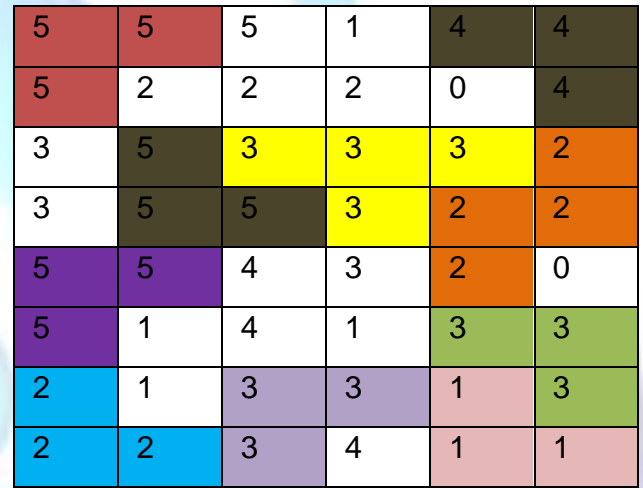

(b)

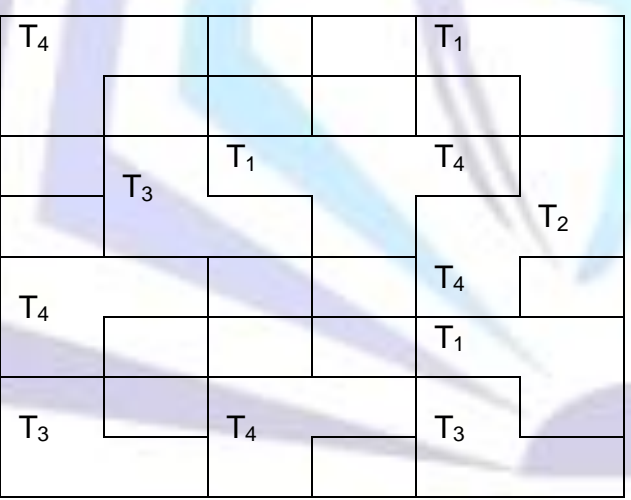

\begin{tabular}{|l|l|l|l|l|l|}
\hline 5 & 5 & 0 & 0 & 4 & 4 \\
\hline 5 & 0 & 0 & 0 & 0 & 4 \\
\hline 0 & 5 & 3 & 3 & 3 & 2 \\
\hline 0 & 5 & 5 & 3 & 2 & 2 \\
\hline 5 & 5 & 0 & 0 & 2 & 0 \\
\hline 5 & 0 & 0 & 0 & 3 & 3 \\
\hline 2 & 0 & 3 & 3 & 1 & 3 \\
\hline 2 & 2 & 3 & 0 & 1 & 1 \\
\hline
\end{tabular}

(d)

Fig. 5: Illustration of the texton detection process (a) Original texture image (b) Transitioned texture image (c) textons names identification (d) Final texton image.

In Fig. 4 the four pixels of a $2 \times 2$ grid are denoted as $V_{1}, V_{2}, V_{3}$ and $V_{4}$. Texton is detected is if threeor more pixels contains the same grey level value. Once the texton $\mathrm{T}_{1}, \mathrm{~T} 2, \mathrm{~T} 3, \mathrm{~T} 4$ and $\mathrm{T}_{5}$ is detected the the proposed texton representation remains the $2 \times 2$ grid as it is without any change. If there are no textons then the $2 \times 2$ grid will be made as zeros. The working mechanism of texton detection is illustrated in Fig.5.

The Texton basedtransitioned LBP texture image is formed by remaining the $2 \times 2$ grid as it is whenever a texton is found otherwise the $2 \times 2$ grid pixels are assigned to zero. This process is repeated starting from top left corner by shifting one column right till it reaches the last column and then shifting bottom by one position till it reaches the last row and last column in a convolution manner.

Step4: In the step four the present paper evaluated the GLCM features on the Texton basedtransitioned LBP texture image. 
One of the most popular statistical methods used to measure the textural information of images is the GLCM. The GLCM method gives reasonable texture information of an image that can be obtained only from two pixels. Grey level cooccurrence matrices introduced by Haralick [37] attempt to describe texture by statistically sampling how certain grey levels occur in relation to other grey levels. Suppose an image to be analyzed is rectangular and has $N_{x}$ rows and $N_{y}$ columns. Assume that the gray level appearing at each pixel is quantized to $N_{g}$ levels. $L_{\text {et }} L_{x}=\left\{1,2, \ldots, N_{x}\right\}$ be the horizontal spatial domain, $L y=\left\{1,2, \ldots, N_{y}\right\}$ be the vertical spatial domain, and $G=\left\{0,1,2, \ldots, N_{g}-1\right\}$ be the set of $N_{g}$ quantized gray levels. The set $L_{x} \times L_{y}$ is the set of pixels of the image ordered by their row-column designations. Then the image I can be represented as a function of co-occurrence matrix that assigns some gray level in $L_{x} \times L_{y} ; \mathrm{I}: L_{x} \times L_{y} \rightarrow G$. The gray level transitions are calculated based on the parameters, displacement $(\mathrm{d})$ and angular orientation $(\theta)$. By using a distance of one pixel and angles quantized to $45^{\circ}$ intervals, four matrices of horizontal, first diagonal, vertical, and second diagonal $\left(0^{\circ}, 45^{\circ}, 90^{\circ}\right.$ and $135^{\circ}$ degrees) are used. Then the un-normalized frequency in the four principal directions is defined by Equation 7 .

$$
\begin{gathered}
p(i, j, d, \theta)=\# \\
\left\{\begin{array}{c}
\left((k, l),(m, n) \epsilon\left|\left(L_{x} \times L_{y}\right) \times\left(L_{x} \times L_{y}\right)\right|\right. \\
(k-m=0,|l-n|=d) \text { or }(k-m=d, l-n=-d) \\
\text { or }(k-m=-d, l-n=d) \text { or }(|k-m|=d, l-n=0), \\
\text { or }(k-m=d, l-n=d) \text { or }(k-m=-d, l-n=-d), \\
I(k, l)=i, \quad I(m, n)=j
\end{array}\right.
\end{gathered}
$$

where \# is the number of elements in the set, $(k, l)$ the coordinates with gray level $i,(m, n)$ the coordinates with gray level $j$. The following Fig. 6 illustrates the above definitions of a co-occurrence matrix $\left(d=1, \theta=0^{0}\right)$ :

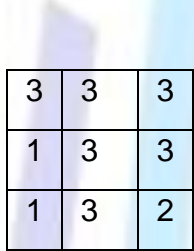

(a)

\begin{tabular}{l|lll}
$0^{\circ 0}$ & 1 & 2 & 3 \\
\hline 1 & 0 & 0 & 2 \\
2 & 0 & 0 & 0 \\
3 & 0 & 1 & 3
\end{tabular}

(b)

\begin{tabular}{l|lll}
$45^{\circ 0}$ & 1 & 2 & 3 \\
\hline 1 & 0 & 0 & 2 \\
2 & 0 & 0 & 0 \\
3 & 0 & 0 & 2
\end{tabular}

(c)

\begin{tabular}{l|lll}
$90^{\circ 0}$ & 1 & 2 & 3 \\
\hline 1 & 1 & 0 & 1 \\
2 & 0 & 0 & 1 \\
3 & 0 & 0 & 3
\end{tabular}

(d)

\begin{tabular}{l|lll}
$135^{\circ} 0^{0}$ & 1 & 2 & 3 \\
\hline 1 & 0 & 0 & 0 \\
2 & 0 & 0 & 1 \\
3 & 0 & 0 & 2
\end{tabular}

(e)

Fig.6: An example of Gray level co-occurrence matrix.

Even though Haralick [37] extracted 24 parameters from co-occurrence matrix, only four are commonly used such as contrast, correlation, energy, homogeneity as given in Equations (8) to (11) and is stored in feature database.

$$
\text { Contrast }=\sum_{i, j=0}^{N-1} P_{i j}(i-j)^{2}
$$

The contrast feature is a difference moment of the $P$ matrix and is a standard measurement of the amount of local variations present in an image. The higher the value of contrast are, the sharper the structural variations in the image.

$$
\text { Correlation }=\sum_{i, j=0}^{N-1} P_{i j} \frac{(i-\mu)(j-\mu)}{\sigma^{2}}
$$

where $\mathrm{P}_{\mathrm{ij}}$ is the pixel value in position (i,j) of the texture image, $\mathrm{N}$ is the number of gray levels in the image, $\mu$ is $\mu=$ $\sum_{i, j=0}^{N-1} i P_{i j}$ mean of the texture image and $\sigma^{2}$ is $\sigma^{2}=\sum_{i, j=0}^{N-1} P_{i j}(i-\mu)^{2}$ variance of the texture image. Correlation is the measure of similarity between two images in comparison.

$$
\text { Energy }=\sum_{i, j=0}^{N-1}-\ln \left(P_{i j}\right)^{2}
$$

Energy measures the number of repeated pairs and also measures uniformity of the normalized matrix.

$$
\text { Homogenity }=\sum_{i, j=0}^{N-1} \frac{P_{i j}}{1+(i-j)^{2}}
$$

It measures the closeness of the distribution of elements in the GLCM to the GLCM diagonal. The converse of homogeneity results in the statement of contrast. 


\section{RESULTS AND DISCUSSIONS}

The proposed TTLBP using GLCM features is experimented on the Tire, Animal fur, Car and Leaf textures collected from Google data base with a resolution of $256 \times 256$. Fig. 7, 8, 9 and 10 shows some of the texture images of Tire, Animal fur, Car and Leaf respectively with their numbers mentioned below the texture images.
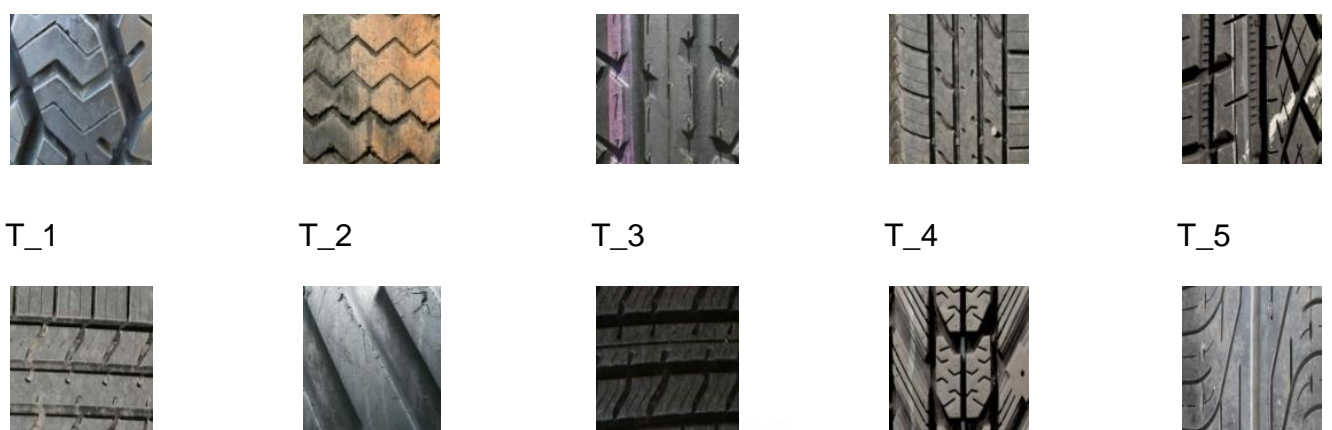

T_2

T_3

T_4

T_5
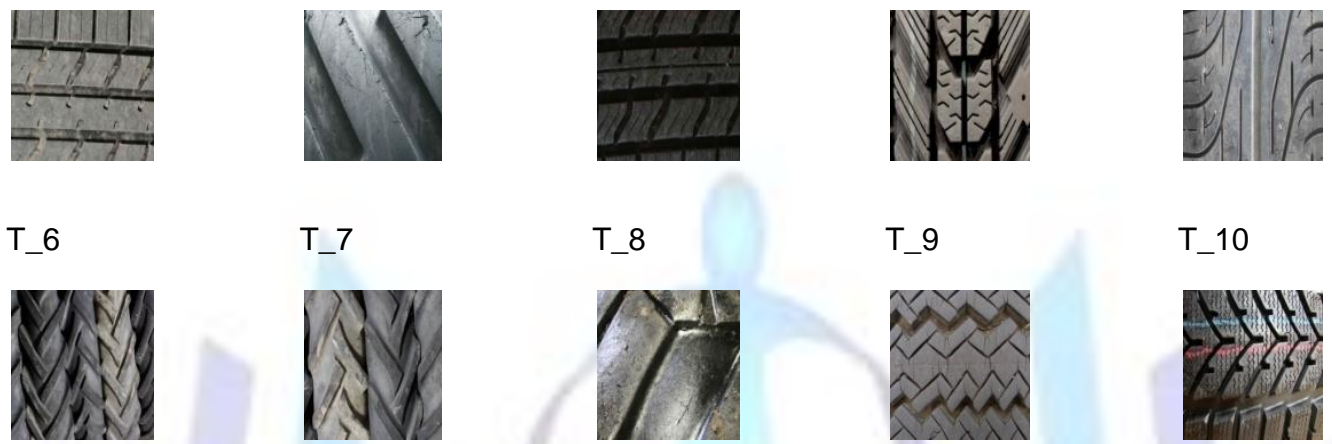

T_7

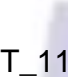

T_12

T_8

T_9

T_10
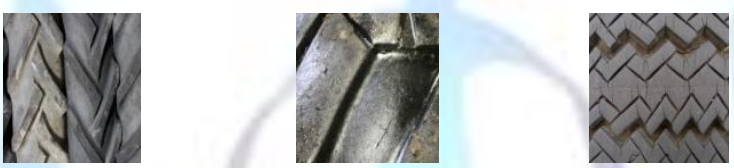

T_13

T_14

Fig.7. Tire texture images.
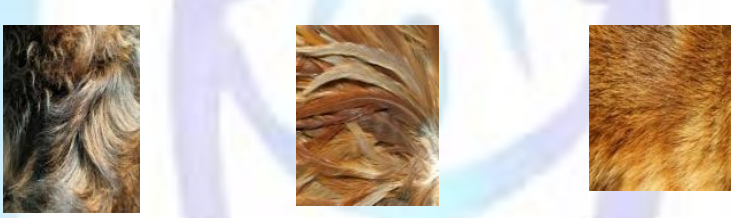

AF_2

AF_3

AF_4
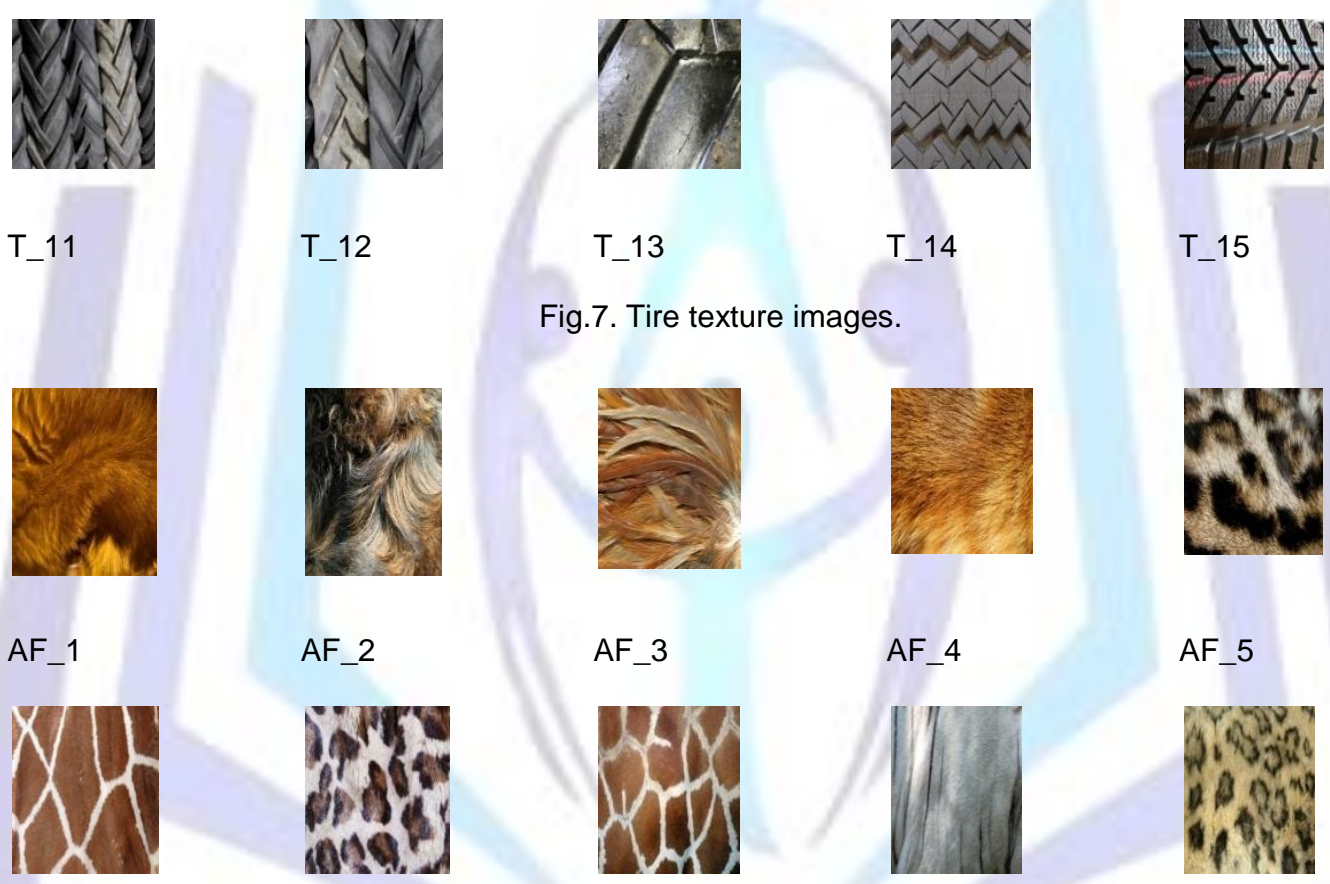

AF 5

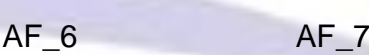

AF_8

AF_9

AF_10
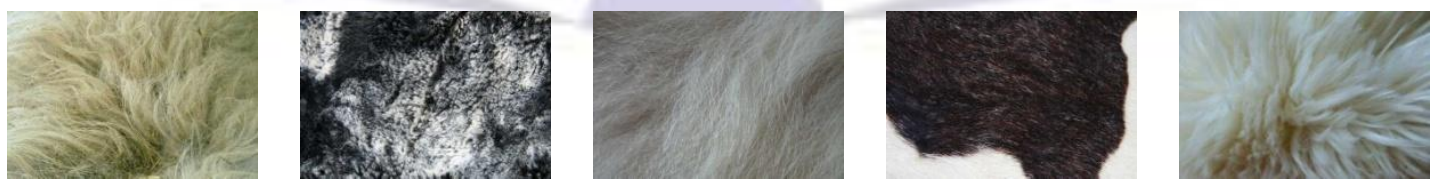
AF_11
AF_12

AF_13

AF_14

AF_15

Fig.8. Animal fur textures images 

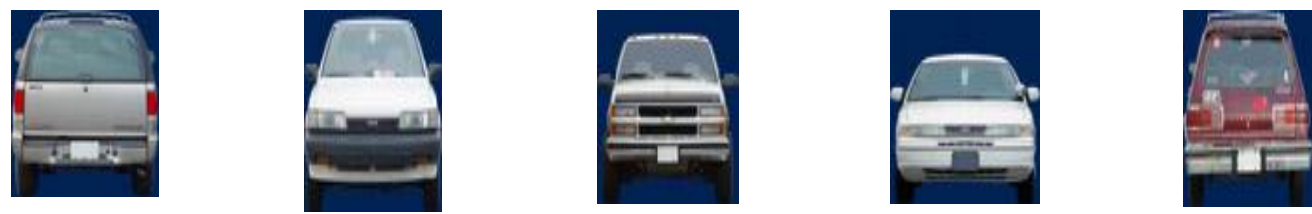

C_1

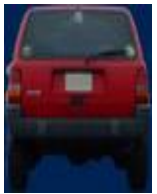

C_6

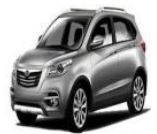

C_11

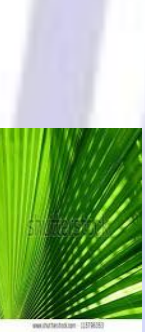

L_1

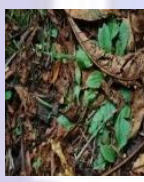

C_2

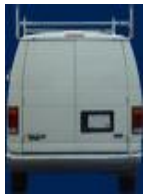

C_7

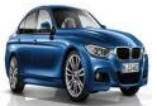

C_12

L_2

L_6

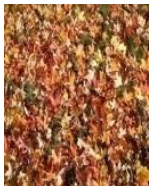

L_11

L_12

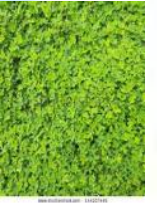

C_3

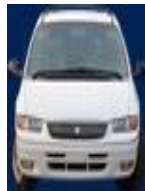

C_8

C_9

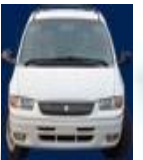

C_13

C_14

Fig.9. Car texture images.

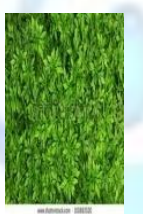

C_4

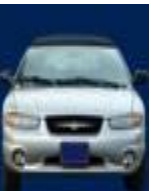

C_5

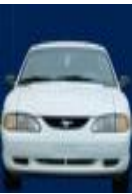

_10

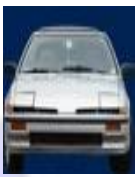

C_15

L_3

L_ 4

L_5

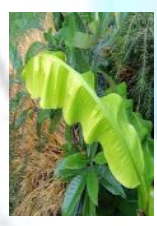

L_8
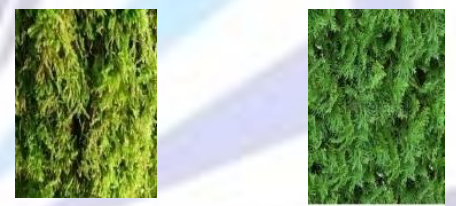

L_9

L_10
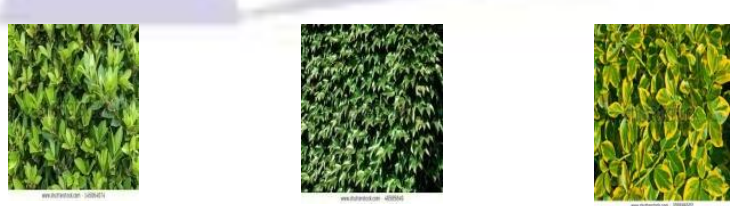

L_13

L_14

L_15

Fig.10: Leaf texture images.

The present paper evaluated the integrated texture features $F_{1}, F_{2}, F_{3}$ and $F_{4}$ using the GLCM features contrast, correlation, energy and homogeneity respectively on TTLBP. The Table 1, 2, 3 and 4 shows the integrated texture features $F_{1}, F_{2}, F_{3}$ and $F_{4}$ evaluated on each of the Animal fur, Tire, Car and Leaf textures respectively using the proposed TTLBP method and placed them in the training data base. Whenever a probe image is given then the integrated texture features $F_{1}, F_{2}, F_{3}$ and $F_{4}$ are evaluated on the probe image using TTLBP,the proposed TTLBP method. Based on the 
nearest neighbourhood the texture features of probe image is compared with training images. The Euclidean minimum distance for image retrieval is calculated by the following equation 12 .

for $\mathrm{j}=1$ to $\mathrm{N}$

$$
d_{j}=\sum_{1}^{4} \sqrt{\left(T_{j} F_{i}-P F_{i}\right)^{2}}
$$

where $\mathrm{N}$ is the number of textures in the data set (training database), the $\mathrm{T}_{\mathrm{j}} \mathrm{F}_{\mathrm{i}}$ refers to theintegrated texture features value of $F_{i}$ for the training texture $T_{j}, P F_{i}$ represents the histogram of the texture feature $F_{i}$ for the probe texture $P$ and $d$ represents the summation of absolute difference between the corresponding texture features of the trained texture $T_{j}$ and probe texture $P$.

The retrieved image $R$ is obtained by the following equation 13

$\mathrm{R}=\min \left(\mathrm{d}_{\mathrm{j}}\right)$ where $\mathrm{j}$ is 1 to $\mathrm{N}$

Based on $\mathrm{R}$ the hit and miss are evaluated and represented in Table 5, 6, 7 and 8 for considered textures. A hit indicates a correct match is found and miss indicates there is miss match between the probe image and image retrieved. In the table $5,6,7$ and 8 hit and miss are shown with binary value 1 and 0 respectively.

Table 1: The texture feature values by the proposed TTLBP method for animal far images.

\begin{tabular}{|c|c|c|c|c|}
\hline Texture No & Contrast $\left(\mathrm{F}_{1}\right)$ & Correlation $\left(\mathrm{F}_{2}\right)$ & Energy $\left(F_{3}\right)$ & Homogeneity $\left(\mathrm{F}_{4}\right)$ \\
\hline Animal_fur_1 & 4.25 & 0.15 & 0.25 & 0.75 \\
\hline Animal_fur_2 & 5.25 & 0.25 & 0.25 & 0.75 \\
\hline Animal_fur_3 & 2.5 & 0.15 & 0.25 & 0.75 \\
\hline Animal_fur_4 & 7.5 & 0.35 & 0.25 & 0.75 \\
\hline Animal_fur_5 & 1.25 & 0.15 & 0.25 & 0.75 \\
\hline Animal_fur_6 & 3.75 & 0.85 & 0.25 & 0.75 \\
\hline Animal_fur_7 & 2.25 & 0.25 & 0.25 & 0.75 \\
\hline Animal_fur_8 & 6.5 & 0.5 & 0.25 & 0.75 \\
\hline Animal_fur_9 & 3 & 0.55 & 0.25 & 0.75 \\
\hline Animal_fur_10 & 7.75 & 0.35 & 0.25 & 0.75 \\
\hline Animal_fur_11 & 2.98 & 0.25 & 0.25 & 0.75 \\
\hline Animal_fur_12 & 1.53 & 0.25 & 0.25 & 0.75 \\
\hline Animal_fur_13 & 4.5 & 0.35 & 0.25 & 0.75 \\
\hline Animal_fur_14 & 5.5 & 0.95 & 0.25 & 0.75 \\
\hline Animal_fur_15 & 6.25 & 0.45 & 0.25 & 0.75 \\
\hline Animal_fur_16 & 2.25 & 0.55 & 0.25 & 0.75 \\
\hline Animal_fur_17 & 6.75 & 0.25 & 0.25 & 0.75 \\
\hline Animal_fur_18 & 4.25 & 0.65 & 0.25 & 0.75 \\
\hline Animal_fur_19 & 3.25 & 0.2 & 0.25 & 0.75 \\
\hline Animal_fur_20 & 1.5 & 0.5 & 0.25 & 0.75 \\
\hline
\end{tabular}


Table 2: The texture feature values by the proposed TTLBP method for Rubber images.

\begin{tabular}{|c|c|c|c|c|}
\hline & Contrast $\left(\mathrm{F}_{1}\right)$ & Correlation $\left(\mathrm{F}_{2}\right)$ & Energy $\left(\mathrm{F}_{3}\right)$ & Homogeneity $\left(\mathrm{F}_{4}\right)$ \\
\hline Rubber_1 & 12.25 & 0.15 & 0.35 & 0.75 \\
\hline Rubber_2 & 10.1 & 0.35 & 0.35 & 0.75 \\
\hline Rubber_3 & 12.3 & 0.45 & 0.35 & 0.75 \\
\hline Rubber_4 & 11.4 & 0.25 & 0.35 & 0.75 \\
\hline Rubber_5 & 10.5 & 0.55 & 0.35 & 0.75 \\
\hline Rubber_6 & 14.8 & 0.65 & 0.35 & 0.75 \\
\hline Rubber_7 & 13.56 & 0.25 & 0.35 & 0.75 \\
\hline Rubber_8 & 12.3 & 0.65 & 0.35 & 0.75 \\
\hline Rubber_9 & 2 & 0.85 & 0.35 & 0.75 \\
\hline Rubber_10 & 14.2 & 0.45 & 0.35 & 0.75 \\
\hline Rubber_11 & 11.1 & 0.35 & 0.35 & 0.75 \\
\hline Rubber_12 & 12.1 & 0.15 & 0.35 & 0.75 \\
\hline Rubber_13 & 8.9 & 0.95 & 0.35 & 0.75 \\
\hline Rubber_14 & 4.6 & 0.35 & 0.35 & 0.75 \\
\hline Rubber_15 & 16.4 & 0.25 & 0.35 & 0.75 \\
\hline Rubber_16 & 17.3 & 0.15 & 0.35 & 0.75 \\
\hline Rubber_17 & 14.5 & 0.25 & 0.35 & 0.75 \\
\hline Rubber_18 & 13.98 & 0.5 & 0.35 & 0.75 \\
\hline Rubber_19 & 10.2 & 0.65 & 0.35 & 0.75 \\
\hline Rubber_20 & 15 & 0.75 & 0.35 & 0.75 \\
\hline
\end{tabular}


Table 3: The texture feature values by the proposed TTLBP method for Leaf images.

\begin{tabular}{|c|c|c|c|c|}
\hline & Contrast $\left(F_{1}\right)$ & Correlation $\left(\mathrm{F}_{2}\right)$ & Energy $\left(\mathrm{F}_{3}\right)$ & Homogeneity $\left(\mathrm{F}_{4}\right)$ \\
\hline leaf_1 & 22.25 & 0.65 & 0.65 & 1 \\
\hline leaf_2 & 21.71 & 0.75 & 0.65 & 1 \\
\hline leaf_3 & 19.75 & 0.64 & 0.65 & 1 \\
\hline leaf_4 & 24.24 & 0.58 & 0.65 & 1 \\
\hline leaf_5 & 26.5 & 0.52 & 0.65 & 1 \\
\hline leaf_6 & 22.1 & 0.79 & 0.65 & 1 \\
\hline leaf_7 & 18.75 & 0.76 & 0.65 & 1 \\
\hline leaf_8 & 27.25 & 0.78 & 0.65 & 0.85 \\
\hline leaf_9 & 28.8 & 0.69 & 0.65 & 1 \\
\hline leaf_10 & 28.5 & 0.87 & 0.65 & 1 \\
\hline leaf_11 & 27.1 & 0.86 & 0.65 & 0.95 \\
\hline leaf_12 & 29.5 & 0.72 & 0.65 & 0.95 \\
\hline leaf_13 & 28.25 & 0.61 & 0.65 & 1 \\
\hline leaf_14 & 25.25 & 0.69 & 0.65 & 1 \\
\hline leaf_15 & 24.25 & 0.73 & 0.65 & 1 \\
\hline leaf_16 & 26.25 & 0.97 & 0.65 & 1 \\
\hline leaf_17 & 21.75 & 0.81 & 0.65 & 1 \\
\hline leaf_18 & 27.25 & 0.73 & 0.65 & 1 \\
\hline leaf_19 & 29.25 & 0.67 & 0.65 & 1 \\
\hline leaf_20 & 24.5 & 0.62 & 0.65 & 0.75 \\
\hline
\end{tabular}

Table 4: The texture feature values by the proposed TCM of TTLBP method for Car images.

\begin{tabular}{|c|c|c|c|c|}
\hline & Contrast $\left(\mathrm{F}_{1}\right)$ & Correlation $\left(\mathrm{F}_{2}\right)$ & Energy $\left(\mathrm{F}_{3}\right)$ & Homogeneity $\left(\mathrm{F}_{4}\right)$ \\
\hline Car_1 & 0.75 & 0.75 & 0.5 & 1 \\
\hline Car_2 & 1 & 0.75 & 0.25 & 1 \\
\hline Car_3 & 1 & 0.5 & 0.25 & 1 \\
\hline Car_4 & 1.25 & 0.75 & 0.25 & 1 \\
\hline Car_5 & 1 & 0.75 & 0.25 & 1 \\
\hline Car_6 & 1.25 & 0.5 & 0.25 & 1 \\
\hline Car_7 & 0.75 & 0.75 & 0.25 & 1 \\
\hline Car_8 & 0.75 & 0.75 & 0.5 & 1 \\
\hline Car_9 & 0.75 & 0.75 & 0.5 & 1 \\
\hline Car_10 & 0.75 & 0.75 & 0.25 & 1 \\
\hline Car_11 & 1 & 0.75 & 0.25 & 1 \\
\hline Car_12 & 1 & 0.75 & 0.25 & 1 \\
\hline Car_13 & 0.75 & 0.75 & 0.25 & 1 \\
\hline Car_14 & 0.75 & 0.75 & 0.75 & 1 \\
\hline Car_15 & 0.75 & & & 1 \\
\hline
\end{tabular}




\begin{tabular}{|c|c|c|c|c|} 
Car_16 & 1.25 & 0.5 & 0.25 & 1 \\
\hline Car_17 & 1.5 & 0.5 & 0.25 & 0.75 \\
\hline Car_18 & 1.75 & 0.5 & 0.25 & 0.75 \\
\hline Car_19 & 1.75 & 0.5 & 0.25 & 0.75 \\
\hline Car_20 & 1 & 0.75 & 0.25 & 1 \\
\hline
\end{tabular}

Table 5: Hit or Miss of Anima fur texture images.

\begin{tabular}{|c|c|}
\hline $\begin{array}{c}\text { Probe } \\
\text { images } \\
\text { (Tire) }\end{array}$ & $\begin{array}{l}\text { Hit/ } \\
\text { Miss }\end{array}$ \\
\hline 1 & 1 \\
\hline 2 & 1 \\
\hline 3 & 1 \\
\hline 4 & 0 \\
\hline 5 & 1 \\
\hline 6 & 1 \\
\hline 7 & 1 \\
\hline 8 & 0 \\
\hline 9 & 1 \\
\hline 10 & 0 \\
\hline 11 & 1 \\
\hline 12 & 0 \\
\hline 13 & 0 \\
\hline 14 & 1 \\
\hline 15 & 0 \\
\hline
\end{tabular}

Table 6: Hit or Miss of rubber texture images.

\begin{tabular}{|c|c|}
\hline $\begin{array}{c}\text { Probe } \\
\text { images } \\
\text { (Animal fur) }\end{array}$ & $\begin{array}{l}\text { Hit/ } \\
\text { Miss }\end{array}$ \\
\hline 1 & 1 \\
\hline 2 & 0 \\
\hline 3 & 1 \\
\hline 4 & 0 \\
\hline 5 & 1 \\
\hline 6 & 1 \\
\hline 7 & 1 \\
\hline 8 & 1 \\
\hline 9 & 0 \\
\hline 10 & 0 \\
\hline 11 & 1 \\
\hline 12 & 1 \\
\hline 13 & 1 \\
\hline 14 & 0 \\
\hline 15 & 1 \\
\hline
\end{tabular}

Table 7: Hit or Miss of leaf texture images.

\begin{tabular}{|c|c|}
\hline $\begin{array}{c}\text { Probe } \\
\text { images } \\
\text { (Car) }\end{array}$ & $\begin{array}{l}\text { Hit/ } \\
\text { Miss }\end{array}$ \\
\hline 1 & 0 \\
\hline 2 & 1 \\
\hline 3 & 1 \\
\hline 4 & 1 \\
\hline 5 & 0 \\
\hline 6 & 1 \\
\hline 7 & 1 \\
\hline 8 & 0 \\
\hline 9 & 1 \\
\hline 10 & 1 \\
\hline 11 & 1 \\
\hline 12 & 0 \\
\hline 13 & 1 \\
\hline 14 & 0 \\
\hline 15 & 1 \\
\hline
\end{tabular}

Table 8: Hit or Miss of car texture images.

\begin{tabular}{|c|c|}
\hline $\begin{array}{l}\text { Probe images } \\
\text { (Leaf) }\end{array}$ & $\begin{array}{l}\text { Hit/ } \\
\text { Miss }\end{array}$ \\
\hline 1 & 1 \\
\hline 2 & 1 \\
\hline 3 & 1 \\
\hline 4 & 0 \\
\hline 5 & 1 \\
\hline 6 & 1 \\
\hline 7 & 0 \\
\hline 8 & 1 \\
\hline 9 & 1 \\
\hline 10 & 0 \\
\hline 11 & 1 \\
\hline 12 & 0 \\
\hline 13 & 1 \\
\hline 14 & 1 \\
\hline 15 & 1 \\
\hline
\end{tabular}

The overall image retrieval performance based on the proposed TTLBP method is shown in Table 9 and it is also shown in the form of bar graph in Fig.11.

Table 9: Retrieval rate of different textures

\begin{tabular}{|l|l|}
\hline Texture Databases & Retrieval rates \\
\hline Animal fur & 60 \\
\hline Rubber & 66.66 \\
\hline Leaf & 66.66 \\
\hline Car & 73.33 \\
\hline Average retrieval rate & $\mathbf{6 6 . 7}$ \\
\hline
\end{tabular}




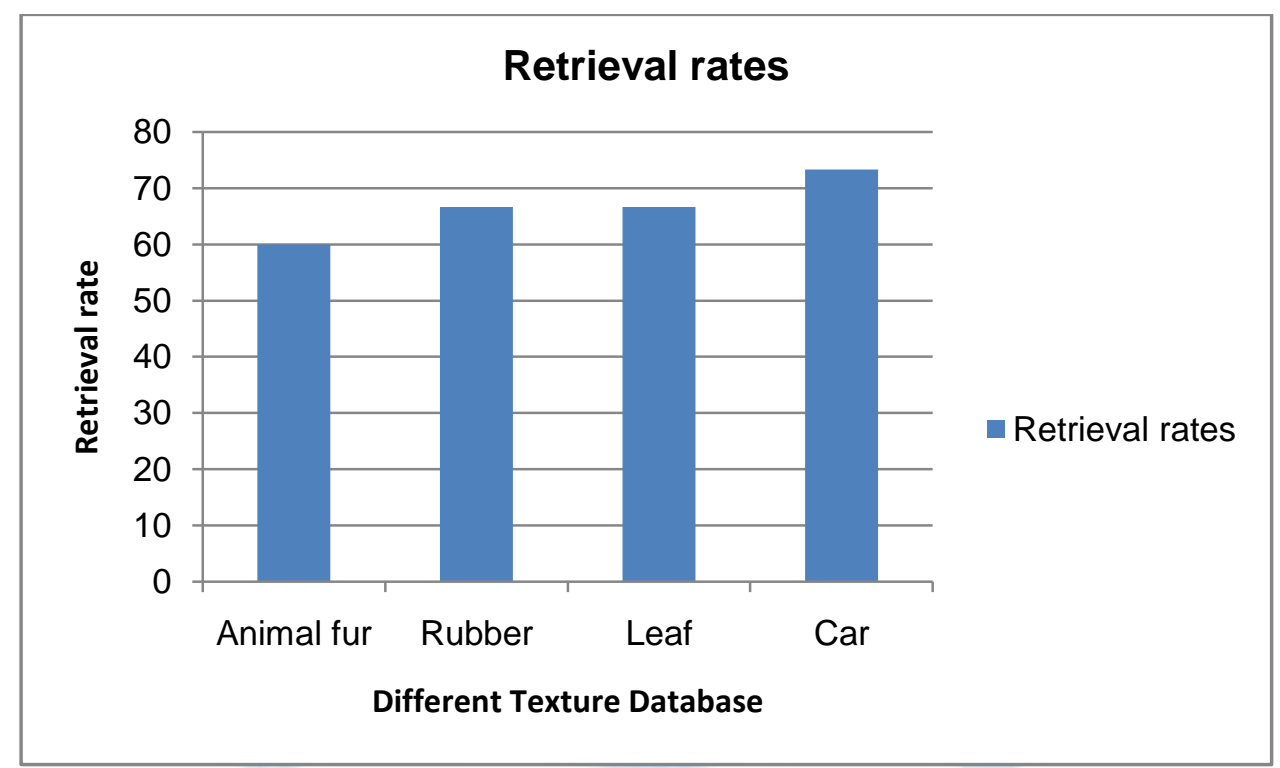

Fig.11: Bar graph representation of retrieval rate of different texture databases.

\section{SUMMARY}

The present paper integrated three popular methods for efficient image retrieval i.e the LBP, textons and GLCM. Initially the present paper derived circular transitions on LBP to achieve rotational invariance and to reduce huge complexity of LBP codes. The main advantage of the present method is, it reduced all the 256 LBP features into 5 features based on the number of transitions. In the second step the textons are evaluated to represent the shape features. In the third step GLCM features are evaluated for efficient image retrieval.

\section{REFERENCES}

[1] EnserP.G.B.“Pictorial information retrieval,” Journal of Documentation, 51(2), 126-170, 1995.

[2] Kato T. "Database architecture for content-based image retrieval" in Image Storage and Retrieval Systems (Jambardino, A A and Niblack, W R, eds), Proc SPIE 1662, 112-123,1992.

[3] Del Bimbo A.Visual Information Retrieval, Morgan Kaufmann (Ed.), San Francisco, USA, 1999.

[4] Goodrum A.A. "Image information retrieval: an overview of current research," Inform. Sci. vol.3(2) pp.63-67, 2000.

[5] Marsicoi L., Cinque and LevialdiS."Indexing pictorial documents by their content: a survey of current techniques," IMC.15 (2) PP. 119-14, 2000.

[6] Rui Y., Huang T.S. and ChangS.F."Image retrieval: current techniques, promising directions and open issues," JVC. Image Rep. vol.10, pp.39-62, 1999.

[7] Smuelders A.W.M, Worring M, Santini. S."Content-based image etrieval at the end of early years", IEEE Trans. PAMI.Vol.22(12) pp.1349-1380, 2000.

[8] Veltkamp R.C. and TanaseM."Content-Based Image Retrieval Systems: A Survey", UU-CS-2000-34,Department of Computer Science, Utretch University, October 2002.

[9] Veltkamp R.C. and Tanase M."A survey of content-based image retrieval systems" in: O. Marques, B.Furht (Eds.), Content-Based Image and Video Retrieval, Kluwer, Dordrecht, 2002.

[10] Prasad B.G., Biswas K.K. and Gupta S.K."Region-based image retrieval using integrated color, shape, and location index," Computer Vision and Image Understanding 9, 193-233, 2004.

[11] Bovik, A. C., Clark M. and Geisler W.S. "Multichannel texture analysis using localized spatial filters," IEEE Trans. Patt. Anal. Mach. Intell., 12, 1, pp. 55-73, 1990.

[12] Flickner M., et al."Query by image and video content: the QBIC system", IEEE Comput. 28 (9) pp.23-32, 1995.

[13] Ma W.Y, and ManjunathB.S."Netra: a toolbox for navigating large image database", Proc. IEEE Int. Conf. on Image Process. 1 pp.568-571, 1997.

[14] Smith J.R and Chang S.F."VisualSEEK: a fully automated content-based image query system," ACM Multimedia , pp.87-98, 1996.

[15] Ogle V.E. and StonebakerM."Chabot: retrieval from a relational database of images", IEEE Comput.28 (9) pp.4048, 1995. 
[16] Carson C., et al."Region-Based Image Querying", in: Proc. CVPR_97 Workshop on Content-based Access to Image and Video libraries (CAIVL_97), 1997.

[17] Pentland A., Picard R.W. and Sclaroff S. "Photobook: Tools for content-based manipulations of image databases, Storage and Retrieval for still Image and video," Databases II ,proc.SPIE２185.IS \& T/SPIE February 1994.

[18] Gudivada V. N. and Raghavan."Content based image retrieval systems," IEEE Comput.28, 9, 18-22.1995.

[19] Kherfi M.L and Ziou D., et al."Image Retrieval From the World Wide Web:Issues, Techniques, and Systems", ACM Surveys, Vol. 36, No. 1, March 2004.

[20] Ratha N. K., Karu K. and Jain A. K. "A real-time matching system for large fingerprint databases,". IEEE Trans. Patt. Analys. Mach. Intell. 18, 8, 799-813.1996.

[21] Martinez A. "Face image retrieval using HMMs". IEEE Workshop CBAI and Video Libraries, pp.25-39.1999.

[22] Gerardts Z. and Bijhold J. "Content based information retrieval in forensic image databases". J. Forensic Sci. 47, 2 , 40-47.2002.

[23] Eakins J.P. and Graham M. E. "Content based image retrieval: A report to the JISC Technology Applications Programme," Institute for Image Data Research, University of Northumbria at Newcastle, Newcastle, U.K.1999.

[24] Schettini R., Ciocca G. and Zuffi S. "Color imaging science: exploiting digital media", in: R. Luo, L. MacDonald, Wiley, New York, 2000 Available from <http://www.citeseer.nj.nec.com/489355.html>.

[25] Hafner J., et al."Efficient color histogram indexing for quadratic form distance functions", IEEE Trans.Pattern Anal. Mach. Intell. 17 (7) 729-736, 1995.

[26] Swain M. and Ballard D. "Color Indexing," Int. Journal of Computer Vision, vol.7, no. 1, pp. 11-32, 1991.

[27] Stricker M., DimaiA."Color indexing with weak spatial constraints", Proc. SPIE, Storage Retrieval Still Image Video Databases IV 2670 pp.29-40, 1996.

[28] Huang J. et al."Image indexing using color correlograms," Internat.Conf. Computer Vision Pattern Recognition pp.762-768, 1997.

[29] Kato T. "Database architecture for content-based image retrieval" in Image Storage and Retrieval Systems (Jambardino, A A and Niblack, W R, eds), Proc SPIE 1662, 112-123,1992.

[30] Zhang H.J., et al, "Image retrieval based on color features: An evaluation study," SPIE Conf. on Digital Storage and Archival, Pennsylvania, Oct. 25-27, 1995.

[31] Kankanahalli M.S. and MehtreB.M."Cluster-based color matching for image retrieval", Pattern Recognition 29 (4), 1996.

[32] Wan X, and Kuo C.J. "A multiresolution color clustering approach to image indexing and retrieval," in: Proc. ICASSP, 1998.

[33] Gonzalez R.C, and WintzP."Digital Image Processing, second ed.," Addison-Wesley, Reading, MA,1987.[A54]

[34] Veltkamp R.C. "Shape matching: similarity measure and algorithms", UU-CS-2001-03, Department of Computer Science, Utretch University, February 2001.

[35] Bovik A., Clark M. and Geisler W.S. "Multichannel texture analysis using localized spatial filters," IEEE Trans. Pattern Anal. 12, pp. 55-73, 1990.

[36] Chang T. and Jay KuoC.C. "Texture analysis and classification with tree-structured wavelet transform," IEEE Trans. Image Process.2 (4), pp. 429-440, 1993.

[37] Haralick R.M., Shanmugam K.K. "Texture features for image classification," IEEE Trans. Syst. Man Cyb. 8 (6), pp. 610-621, 1973.

[38] Manjunath B.S. and Ma W.Y."Texture features for browsing and retrieval of image data," IEEE Trans. Pattern Anal. 18 (8), pp. 837-842, 1996.

[39] Raghu P.P. and YegnanarayanaB."Segmentation of Gabor filtered textures using deterministic relaxation," IEEE Trans. Image Process. 5 (12), pp. 1625-1636, 1996.

[40] Raju U. S. N. and Nagaraja Rao A. etal."A new texture segmentation method using with direction measures," Proceedings of NVGIP-05, JNNCE, Karnataka, pp. 107, 2-3 March-2005.

[41] Raju U. S. N. and Vijaya Kumar V. et al. "Texture segmentation using wavelet decomposition," Proceedings of JTalent, Jyothishmathi College of Engineering and Technology, Hyderabad, pp. 28, $2^{\text {nd }}$ and $3^{\text {rd }}$ Feb-2007.

[42] Raju U. S. N. and Vijaya Kumar V. etal."Pipeline Implementation of New Segmentation Based on Cognate Neighborhood Approach,” IAENG, Vol. 35, Issue 1, pp. 1-6, 2008. 
[43] Raju U. S. N. and Vijaya Kumar V. etal."Texture Segmentation Methods Based on Combinatorial of Morphological and Statistical Operations,” JM, Vol.3, No.1, pp. 36-40, 2008.

[44] Teuner A., Pichler O. and HosfickaB.J.“Unsupervisedtexture segmentation of images using Tuned matched Gabor filters," IEEE Trans. Image Process. 4 (6), pp. 863-870, 1995.

[45] Unser M.“Local linear transforms for texture measurements," Signal Process. 11, pp. 61-79, 1986.

[46] Unser M.“Texture classification and segmentation using wavelet frames," IEEE Trans. Image Process. 4 (11), pp. 1549- 1560, 1995.

[47] U Ravi Babu, Vakulabharanam.Vijayakumar B Sujatha, "Texture Classification Based on Texton Features", International Journal of Image, Graphics and Signal Processing (IJIGSP),Vol.4, No.8, August 2012.

[48] Vakulabharanam Vijaya Kumar et.al," Texture Classification based on Texton Patterns using on various Grey to Grey level Preprocessing Methods ", International Journal of Signal Processing, Image Processing and Pattern Recognition, Vol. 6, No. 4, August, 2013.

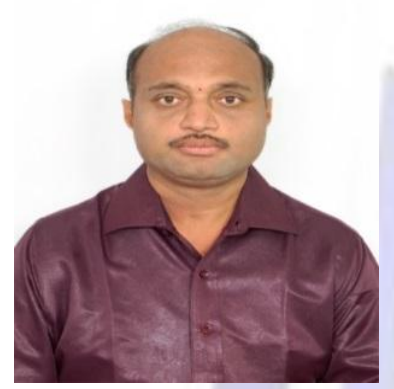

He is presently working as Principal (i/c)\&Assoc.professor, Head; C.Sc.Dept Montessori Siva Sivani institute of Science \& Technology College of Engineering Mylavaram. As a Research scholar pursuing my Ph.d from Krishna university, Machalipatnam under the eminent guidance of Prof.V.Venkata Krishna. As an Academician 19 Years of experience teaching to UG and PG students of Computer Applications \& Sciences. Besides 6 years of Industrial experience as a freelance programmer. Examiner for conducting practical's, paper valuations under Acharya Nagarjuna University, S.V. University, Kakatiya University, IGNOU University, Krishna University and JNTU (K).he is Organizing, Advisory member for various National, International Conferences in the field of Information Technology. Member in various Professional Bodies like ISPACE, ASCAP, IACSIT etc.

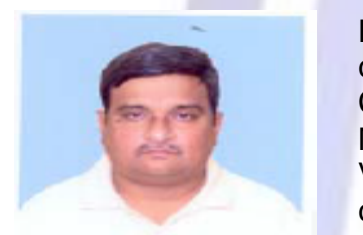

Dr V Venkata Krishna received the B.Tech. (ECE) degree from Sri VenkateswaraUniversity. He completed hisM. Tech. (Computer Science) from JNT University. He received his Ph.D in Computer Science from JNT University in 2004. He worked as Professor and Head for ten years in Mahatma Gandhi Institute of Technology, Hyderabad. After that he worked as Principal for VidyaVikas College of Engineering, Hyderabad for two years. Then he worked as Principal for chaitanya Institute of Science and Technology, JNTU, Kakinada, India for one year. Presently he is working as Principal in Chaintanya Institute of Engineering and Technology, JNTU, Rajahmundry, India. He is an advisory member for many Engineering colleges. He has published 25 research articles. Presently he is guiding 10 research scholars. He is a life member of ISTE and CSI.

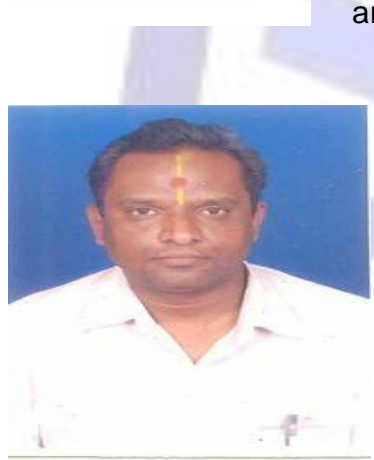

Y. K. Sundara Krishna qualified in Ph.D. in Computer Science \& Engineering from Osmania University, Hyderabad. Now, he is working as Professor in the Department of Computer Science, Krishna University, and Machilipatnam. His research interests are Mobile Computing, Service Oriented Architecture and Geographical Information Systems and having practical work experience in the areas of Computing Systems including Developing of Simulators for Distributed Dynamic Cellular Computing Systems, Applications of Embedded \& Win32 clients, Maintenance of Multi-user System Software. Also he is working with International Telecommunications Union (ITU): Y. 2018 recommendation series Y: Global Information Infrastructure, Internet Protocol aspects and NGN. 